Meta

Journal des traducteurs

Translators' Journal

\title{
À propos de la traduction du " Tombeau des rois "
}

\section{Pierre Marchand}

Volume 21, numéro 2, juin 1976

URI : https://id.erudit.org/iderudit/003558ar

DOI : https://doi.org/10.7202/003558ar

Aller au sommaire du numéro

Éditeur(s)

Les Presses de l'Université de Montréal

ISSN

0026-0452 (imprimé)

1492-1421 (numérique)

Découvrir la revue

Citer cet article

Marchand, P. (1976). À propos de la traduction du « Tombeau des rois ». Meta, 21(2), 155-160. https://doi.org/10.7202/003558ar d'utilisation que vous pouvez consulter en ligne.

https://apropos.erudit.org/fr/usagers/politique-dutilisation/ 


\section{À PROPOS DE LA TRADUCTION DU « TOMBEAU DES ROIS »}

C'est avec intérêt que nous avons lu dans Meta, vol.xx, $n^{\circ} 3$, le compte rendu de Jean Delisle sur le Dialogue sur la traduction, à propos du Tombeau des rois. L'idée principale qui se dégage de l'article se formule de la façon suivante :

Le dialogue qui s'est établi en 1959 sous forme d'échange de lettres entre les deux poètes démontre de façon éclatante que la traduction, loin de dénaturer ou travestir un poème, lui donne un nouveau visage, le fait briller d'un nouvel éclat ${ }^{1}$.

1. Jean Delisle, Meta, vol. $\mathrm{xx}, \mathrm{n}^{\circ}$ 3, p. 228. 
La lecture du Dialogue sur la traduction nous a amené, quant à nous, à des conclusions différentes et nous tenterons ici de montrer pourquoi. La cinquième partie de l'ouvrage en question contient un commentaire détaillé de la première traduction du Tombeau des rois; c'est là qu'Anne Hébert et Frank Scott « dialoguent $\gg$ sur la traduction du poème. Anne Hébert fait au total 37 commentaircs. Sur ce nombre, 33 concernent des questions sémantiques et posent la question suivante : tel mot anglais rend-il bien le sens de tel mot français? Il est important de garder en mémoire cette préoccupation à peu près exclusivement sémantique (le mot est à prendre ici dans un sens restreint et désigne l'aspect conceptuel du signe).

D'autre part, Anne Hébert donne deux jugements d'ensemble sur la traduction de son poème. Le premier sanctionne la légitimité de la traduction de la poésie et s'appuie, pour ce faire, sur un critère d'utilité secondaire :

Et si j'ai pu vous expliquer certaines alliances de mots et d'images, certaines particularités de langage, votre vive attention, vos questions précises, certains malentendus de langue éclaircis m'ont souvent permis d'approfondir le sens caché de quelques passages ${ }^{2}$.

Dans cette phrase, l'intérêt que manifeste le poète pour la traduction de son œuvre ne s'explique pas par la qualité même du travail du traducteur mais bien par le fait que la traduction pose des difficultés qui obligent l'esprit à serrer le texte de près afin de pouvoir le traduire. La traduction offre en somme l'avantage accidentel de permettre l'approfondissement du texte original.

Cependant, Anne Hébert porte un second jugement qui se révèle beaucoup plus important puisqu'il concerne la qualité même du travail de Frank Scott :

Cher Frank Scott, je m'excuse de ces notes grammaticales si méticuleuses et quelque peu mesquines. Toutes ces pauvres remarques n'entament en rien, je vous assure, l'admiration que j'ai pour votre texte, pour son extraordinaire fidélité grammaticale et sa correspondance presque totale à mon poème ${ }^{3}$.

Voilà un jugement d'ensemble très favorable de la part d'un poète sur un autre poète qui a fait œuvre de traduction. Les deux termes qu'il faut retenir sont «fidélité » et «correspondance ». Ils indiquent qu'Anne Hébert juge la version anglaise comme étant une reproduction de la version française. Il apparât ici que le mot «grammatical » prend un sens particulier, étant donné les préoccupations presque exclusivement sémantiques qui marquent le commentaire de l'auteur du Tombeau des rois : le mot renvoie, semble-t-il, au domaine de la lexicologie, de la sémantique, de la stylistique mais non à celui de la phonétique, encore moins à celui de la phonologie. Et c'est là, croyons-nous, que se situe la faille du dialogue. En effet, sur les 37 commentaires d'Anne Hébert, quatre seulement concernent l'aspect phonique de l'œuvre littéraire, et ces quatre commentaires sont défavorables. Nous en citons un, à titre d'exemple :

2. Anne Hébert et Frank Scott, Dialogue sur la traduction, Montréal, Editions HMH, «Sur parole», 1970, p. 49.

3. Ibid., p. 86 . 
L'immobile désir des gisants me tire. Il y a ici un jeu et un retour de son $i$ : immobile désir des gisants me tire et des sons $e$ et $e ́$, ainsi que des consonnes liquides $l$ et $r$ ainsi que des consonnes continues : gisant, désir. Tout cela n'est guère traduisible. C'est pourquoi il n'y a pas de correspondance sensible entre L'immobile désir et The motionless desire ${ }^{4}$.

Anne Hébert n'ignore donc pas qu'il peut être impossible d'établir une correspondance sensible entre un vers français et sa traduction. Le texte que nous venons de lire contient les mots les plus importants de tout le Dialogue sur la traduction, puisqu'il révèle une préoccupation commune à tous les poètes : celle de la perception sensible de la poésie.

Nous voilà donc devant une ambiguilté : dans l'ensemble, Anne Hébert juge favorablement la traduction de son poème et il est permis de penser que ce jugement concerne l'aspect sémantique de son œuvre ; d'autre part, le poète, dans ses rares commentaires d'ordre phonétique, formule des jugements défavorables. Avant d'aller plus loin, voyons ce qu'il en est de l'argumentation de Frank Scott :

The original poem is itself a translation into a chosen language of that inner stirring of emotion and thought which started the poet on the act of creation. A poem is conceived before it can be percieved. [...] In one sense, even the reading of a poem is a form of translation, for the reader must extract its meaning and significance. Without changing the words, he fills them with his own content, which is not necessarily that of the poet. At another time he will find other meanings ${ }^{5}$.

Le problème est de savoir si l'on peut véritablement parler du projet d'un poète, c'est-à-dire d'une idée qui existerait avant le poème lui-même. Ainsi posée, la question, croyons-nous, trouve facilement réponse : le poème ne peut se concevoir en dehors de son exécution. Frank Scott, quant à lui, insiste beaucoup sur l'existence d'un à priori à l'acte poétique.

La seconde idée avancée par Frank Scott assimile la lecture à une forme de traduction : lorsque nous lisons, nous interprétons l'œuvre en lui donnant des contenus différents de ceux qu'avait prévus le poète et même différents de ceux que nous avons trouvés dans nos lectures antérieures. Il est intéressant de noter ici que l'idée de traduction appelle celle de la différence et non pas de la ressemblance des contenus.

Les deux aspects de l'argumentation de Frank Scott se rejoignent donc en s'appuyant sur une même donnée : l'universalité de la traduction; le poète traduit une idée en poème et le lecteur traduit le poème en l'interprétant. La conclusion vient d'elle-même : si l'acte poétique et la lecture sont des traductions, il n'existe aucun obstacle à la traduction en langue étrangère. Pour Frank Scott, il y a une différence de degré et non de nature entre l'interprétation d'un poème et sa traduction en langue étrangère. Les lignes qui suivent tendent à confirmer l'existence d'une telle conclusion :

4. Dialogue sur la traduction, p. 71.

5. Ibid., p. 94-95. 
The translator [...] must stand back from his sketch to see it from a distance before the imbalance of the parts to the whole can be detected. This of course is true of the original poem, which the poet himself certainly revises as he composes, and may revise again in later publication ${ }^{6}$.

Mais il faut se demander si le traducteur et le poète révisent leur travail pour les mêmes raisons. Le traducteur ne peut éviter le modèle qu'il a sous les yeux, en l'occurrence le texte à traduire. C'est pourquoi l'idée d'un à priori à l'acte poétique est apparue chez Frank Scott : si le traducteur doit réviser son travail afin de le rapprocher de son modèle, pourquoi n'en serai-il pas ainsi du poète qui révise son travail ?

En fait, l'analogie ne tient pas. Les incessantes retouches pratiquées par le poète n'ont pour but que de rendre l'ouvre davantage semblable à elle-même et non pas à un concept qu'il faut traduire. Si le traducteur a un modèle, le poète, lui, ne peut compter que sur son propre poème. C'est pourquoi la traduction d'un poème ne saurait être une étape additionnelle dans la chaîne des traductions engendrées par une idée ou une émotion initiales. Il y a une différence de nature entre l'acte d'écrire un poème et celui de le traduire.

Cependant, pas plus qu'Anne Hébert, Frank Scott n'escamote l'aspect sensible - phonique - de la poésie. Le Dialogue sur la traduction l'indique en plusieurs circonstances et il n'en faut pour preuve que cette phrase : "The translator must hear as well as understand the original ${ }^{7}$.

Il faut donc en arriver à la conclusion suivante : contrairement à ce qui était d'abord apparu, Anne Hébert et Frank Scott ne font pas un sort différent à la Voix et à la Pensée, pour reprendre les termes de Valéry. S'il existe un hiatus, il ne concerne pas la conception que les deux poètes se font de la poésie. La divergence semble plutôt apparaître entre, d'une part, les considérations des deux poètes sur la poésie ( correspondance sensible» - «the translator must hear...») et, d'autre part, les arguments qui tentent de sanctionner la traduction poétique.

Le problème apparaît donc clairement : c'est le désir de cautionner la traduction de la poésie qui a amené les deux poètes à scinder leur argumentation. Anne Hébert et Frank Scott ont fait comme tout le monde et ils ont abordé la question de la traduction en se situant dans une perspective sémantique. Mais ils ont bien senti l'insatisfaction profonde qu'ont provoquée en eux les questions d'ordre phonique.

Après avoir fait ressortir les contradictions qui marquent les prises de position d'Anne Hébert et de Frank Scott, il serait intéressant de comparer le Tombeau des rois avec sa version anglaise. Nous ne voudrions pas reprendre dans sa totalité l'étude que nous avons effectuée il y a quelques années. Qu'il suffise de dire dans un premier temps que le poème d'Anne Hébert exprime un déchirement qui se manifeste de bien des façons. Tout est opposition dans le poème. Nous nous contenterons de donner la liste des oppositions avec des exemples dans chaque cas.

6. Dialogue sur la traduction, p. 94.

7. Ibid., p. 79. 
Opposition des sonorités et du sémantisme :

Opposition des sonorités entre elles :

Opposition des aspects sémantiques :

Opposition des rythmes :

Opposition du rythme et du sémantisme :
Sonorités heurtées évoquant des bruits de la vie quotidienne, comme le gazouillis d'un enfant (ex: au v. $10:$ : Au long des dédales sourds $\gg \rightarrow$ de, de, da).

Contexte sémantique surnaturel, hiératique, onirique, mythologique : «les tombeaux des rois», «fil d'Ariane », "fumée d'encens».

V. 43 à 46 où l'on trouve exactement 9 orales $(2, a, \alpha)$ et 9 nasales $(y, a)$.

V. 57 qui présente une difficulté de lecture ( $\ll$ main sèche qui cherche $\gg)$

V. 27 : «noirs ossements 》.

V. 29 《tragédies patiemment travaillées ».

V. $29-33:$ : tragédies [...] sans larmes ni regrets 》.

V. $58:$ : livide et repue ».

V. 40 : «L'ombre de l'amour/me maquille à petits traits précis » (début lent et fin précipitée).

V. 48 et 49 : a Simulant le dernier tourment

Cherchant son apaisement * (le rythme marque une cadence joyeuse et contredit le sémantisme somme toute assez grave des deux vers).

Toutes ces oppositions, dont nous ne détaillons pas ici l'explication, contribuent à former deux mondes que l'on peut représenter ainsi :

\section{MONDE DE L'ENFANCE \\ ET DU QUOTIDIEN}

Contexte phonique : bruits quotidiens

(plus les difficultés de lecture).

Voyelles orales.

Mots concrets, mots quotidiens.

Rythmes saccadés.

Rythme joyeux.

\author{
MONDE ONIRIQUE, HIÉRATIQUE, \\ SURNATUREL ET ADULTE \\ Contexte sémantique : rêve, mort, rites \\ sacrés, noblesse. \\ Voyelles nasales. \\ Mots recherchés, mots abstraits. \\ Rythmes allongés. \\ Contexte sémantique de l'inquiétude.
}

Retrouve-t-on pareille opposition dans la traduction anglaise ${ }^{8}$ ? Nous ne le pensons pas. Il ne saurait être question de reprendre notre argumentation dans ses moindres détails. Nous nous contenterons de quelques remarques données à titre d'exemple.

Si l'on compare avec l'anglais les vers français contenant, sur le plan phonique, les caractéristiques décrites plus haut, l'on s'aperçoit que la traduction n'a pu tenir compte ni des sonorités concrètes du texte original ni des particularités du rythme. L'exemple le plus frappant est le vers 57 : à " la main sèche qui cherche » correspond «the dried hand seeking ». Deux impressions s'opposent ici ; d'une part, un certain malaise attribuable à la difficulté de lire les mots français et, d'autre part, un certain plaisir engendré par la fluidité de la tournure anglaise. Ajoutons que nous n'avons pas trouvé, dans la proximité du vers 57 , une tournure anglaise qui aurait pu compenser ce que nous considérons comme un changement de tonalité.

Or, un tel changement de tonalité ne constitue pas une exception dans la version anglaise du Tombeau des rois. Pour nous en convaincre, nous avions tenté une petite expérience qui, pour n'avoir rien de scientifique, nous a semblé néanmoins révélatrice. En faisant lire, par la même personne, le poème original et sa

8. Il s'agit de la troisième version consécutive au dialogue entre les deux poètes. 
traduction, nous avons cru remarquer que la narratrice a changé d'attitude lorsqu'elle est passée du français à l'anglais. D'une part, son ton, de dur et sévère qu'il était, est devenu beaucoup plus « doux » en anglais ; d'autre part, la lectrice avait trébuché sur le vers 57 ce qui, on s'en doute bien, ne fut aucunement le cas en anglais. Le phénomène que nous avons observé, même s'il est subjectif - et peutêtre précisément parce qu'il l'est - est révélateur de l'impression que produit un poème, ou toute œuvre littéraire ou non, sur celui qui le lit. Or, si l'impression change lorsqu'on lit la traduction de l'ouvre, peut-on vraiment parler de «correspondance presque totale $\gg$ ?

Nous n'avons donc pas retrouvé, pour notre part, les caractères phoniques si particuliers du poème original. Il est évident que la version anglaise peut, sur le plan sémantique, rendre compte des notations de déchirement, de séparation qui, en français, confirment l'opposition du niveau phonique et d'un certain aspect du niveau sémantique. Mais précisément à cause de ses caractéristiques sonores et rythmiques, le poème anglais tend vers l'harmonie et le raffinement alors que l'œuvre originale tend à nier constamment, par des voies détournées, tout ce qu'elle peut contenir de sacré.

La question est donc de savoir si Frank Scott aurait pu reproduire de façon différente, mais équivalente, les caractéristiques sonores de l'œuvre originale. $\mathrm{Ou}$, au contraire, ne pouvait-il faire autrement que de suivre la voie qu'il a adoptée, étant donné les caractères inévitablement différents de l'anglais?

Après avoir vu toutes les difficultés qu'ont soulevées les différents aspects phoniques de l'œuvre à traduire, il semble bien qu'il soit impossible de parler de traduction, au sens où l'on entend généralement ce mot. La différence des caractéristiques phoniques des langues ne peut qu'engendrer des poésies radicalement différentes et indomptables.

Pourquoi alors des poètes comme Anne Hébert et Frank Scott ont-ils sanctionné l'idée d'une traduction de la poésie ? Il est difficile de le savoir. Nous pensons, pour notre part, que la traduction poétique constitue un moyen efficace de faire connaître l'existence d'une ouvre que la «barrière des langues » rend inaccessible à beaucoup. Cette idée rejoint celle de l'utilité secondaire dont nous parlions au début de la lettre. Nous ne voulons pas nier l'utilité ni même la nécessité de traduire les œuvres poétiques. Nous voulons simplement remettre à sa place - humble mais non inexistante - tout essai de traduction de la poésie, le but ultime de cet exercice devant être d'inspirer au lecteur le désir de prendre connaissance de l'œuvre originale.

Pierre Marchand

P.S. : Ceux qui s'intéressent à la question sont priés de lire l'intéressant article que Noël Audet a fait paraître dans Voix et images, vol. $1, n^{\circ} 1$, et qui s'intitule "Poèmes des quatre côtés de Jacques Brault ». 